TRANSACTIONS OF THE

AMERICAN MATHEMATICAL SOCIETY

Volume 358, Number 2, Pages 893-910

S 0002-9947(05)03717-7

Article electronically published on April 22, 2005

\title{
REPRESENTATION FORMULAE AND INEQUALITIES FOR SOLUTIONS OF A CLASS OF SECOND ORDER PARTIAL DIFFERENTIAL EQUATIONS
}

\author{
LORENZO D'AMBROSIO, ENZO MITIDIERI, AND STANISLAV I. POHOZAEV
}

\begin{abstract}
Let $L$ be a possibly degenerate second order differential operator and let $\Gamma_{\eta}=d^{2-Q}$ be its fundamental solution at $\eta$; here $d$ is a suitable distance. In this paper we study necessary and sufficient conditions for the weak solutions of $-L u \geq f(\xi, u) \geq 0$ on $\mathbb{R}^{N}$ to satisfy the representation formula
\end{abstract}

$$
u(\eta) \geq \int_{\mathbb{R}^{N}} \Gamma_{\eta} f(\xi, u) d \xi
$$

We prove that $(\mathrm{R})$ holds provided $f(\xi, \cdot)$ is superlinear, without any assumption on the behavior of $u$ at infinity. On the other hand, if $u$ satisfies the condition

$$
\liminf _{R \rightarrow \infty} f_{R \leq d(\xi) \leq 2 R}|u(\xi)| d \xi=0
$$

then $(\mathrm{R})$ holds with no growth assumptions on $f(\xi, \cdot)$.

\section{INTRODUCTION}

In the recent paper [12] we studied the following challenging problem. Consider a pair $(L, f)$, where $L$ is a linear differential operator and $f$ a given nonnegative Caratheodory function, that is,

$$
f: \mathbb{R}^{N} \times \mathbb{R} \rightarrow \mathbb{R}
$$

satisfies

i) $s \rightarrow f(\xi, s)$ is continuous for almost every $\xi \in \mathbb{R}^{N}$,

ii) $\xi \rightarrow f(\xi, s)$ is measurable for all $s \in \mathbb{R}$.

Suppose that the set of distributional solutions of

$$
L(u) \geq f(x, u) \quad \text { on } \quad \mathbb{R}^{N}
$$

is not empty and that no information on the solutions at infinity is known.

What are the assumptions on the pair $(L, f)$ that guarantee the inverse positivity of $L$ on the set of solutions of (1.1)?

It turns out that a good and classical way for giving an answer to this problem is to find representation formulas (inequalities) for the solutions of (1.1). However, since no decay on $u$ at infinity is assumed, there is no short-cut to give an answer to this problem by using comparison principles.

Received by the editors April 19, 2004

2000 Mathematics Subject Classification. Primary 35H10, 35C15, 26D10.

(C)2005 American Mathematical Society 
On the other hand, in general, the answer to the problem is negative as the following simple example shows.

Consider

$$
-\Delta u \geq|u|^{p} \quad \text { on } \quad \mathbb{R}^{N}
$$

where $0<p \leq 1$. It is easy to see that, for any $p$ with $0<p \leq 1$, the function defined by

$$
u(x)=-e^{x_{1}^{2}}
$$

is a regular solution of (1.2). Indeed, the results of [12] show that the main ingredient for solving the problem in the affirmative is the superlinearity of the function $f$.

In this paper we address the question of finding representation formulae for solutions of semilinear equations or inequalities associated to a general class of differential operators. In particular we can handle nonlinear problems associated to the Kohn laplacian on the Heisenberg group or, more generally, differential inequalities on Carnot groups. We are especially motivated to consider our problems on the latter structure because Carnot groups arise in several contexts: optimal control theory, asymptotic geometry of manifold with negative curvature, CR geometry and study of hypo-elliptic partial differential equations. Roughly speaking, all results in this paper are essentially proved by using a classical tool, namely judicious choice of test functions.

The paper is organized as follows. The main body of the next section is devoted to the formulation and proof of the representation formulae for various types of semilinear equations and inequalities under different assumptions on the nonlinearity and on the possible solutions that we are dealing with. In particular we prove (see Corollary 2.10 below) a nonexistence result for semilinear inequalities associated to sublinear nonlinearities.

Subsection 2.1 deals with some remarks on representation of solutions of systems of semilinear inequalities. Section 3 contains preliminary facts on Carnot groups and to the formulation of related representation formulae for solutions of semilinear problems. In particular we consider problems on the Heisenberg group associated to the Kohn Laplacian. We end the paper with a note on problems involving Grushin type operators.

The results contained in this paper have been announced at the International INdAM meeting Nonlinear Partial Differential Equations and connected Geometrical Problems, September 2-4, 2003, Grado, Italy.

1.1. Notation. The symbol $\|\cdot\|_{\infty}$ stands for the norm in $L^{\infty}\left(\mathbb{R}^{N}\right)$, while for $\xi \in$ $\mathbb{R}^{N},|\xi|$ denotes the Euclidean norm of $\xi$. For $p>1$ we denote by $p^{\prime}$ its conjugate, that is, $\frac{1}{p}+\frac{1}{p^{\prime}}=1$. For any measurable set $E \subset \mathbb{R}^{N}$, its measure is indicated by $|E|$.

\section{MAin RESULTS}

Let $\mu \in \mathscr{C}^{1}\left(\mathbb{R}^{N} ; \mathbb{R}^{l}\right)$ be a matrix with entries, $\mu:=\left(\mu_{i j}\right), i=1, \ldots, l, j=$ $1, \ldots, N$. Let $X_{i}(i=1, \ldots, l)$ be the vector field defined by

$$
X_{i}:=\sum_{j=1}^{N} \mu_{i j}(\xi) \frac{\partial}{\partial \xi_{j}}
$$


and let $\nabla_{L}$ be defined by

$$
\nabla_{L}:=\left(X_{1}, \ldots, X_{l}\right)^{T}=\mu \nabla .
$$

We shall denote by $X_{i}^{*}$ the formal adjoint of $X_{i}$, that is,

$$
X_{i}^{*}:=-\sum_{j=1}^{N} \frac{\partial}{\partial \xi_{j}} \mu_{i j}(\xi),
$$

and set $\nabla_{L}^{*}:=\left(X_{1}^{*}, \ldots, X_{l}^{*}\right)^{T}$.

Let $L$ be a second order differential operator defined by

$$
L:=-\sum_{i=1}^{l} X_{i}^{*} X_{i}=-\nabla_{L}^{*} \cdot \nabla_{L} .
$$

We shall assume that

$$
\sum_{j=1}^{N} \frac{\partial}{\partial \xi_{j}} \mu_{i j}(\xi)=0, \quad i=1, \ldots, l .
$$

Notice that in this case $\nabla_{L}^{*}=-\nabla_{L}$ and $L=\sum_{i=1}^{l} X_{i}^{2}=\nabla_{L} \cdot \nabla_{L}$.

Definition 2.1. Let $\eta \in \mathbb{R}^{N}$ be fixed. We say that $\left(\mathbf{H}_{\eta}\right)$ is satisfied if there exist a real number $Q=Q(\eta)>2$ and a nonnegative continuous function $d_{\eta}: \mathbb{R}^{N} \rightarrow \mathbb{R}_{+}$ such that the following four properties hold:

(1) $d_{\eta}(\xi)=0$ if and only if $\xi=\eta$.

(2) $d_{\eta} \in \mathscr{C}^{2}\left(\mathbb{R}^{N} \backslash\{\eta\}\right)$.

(3) The fundamental solution of $-L$ on $\mathbb{R}^{N}$ at $\eta$ is given by $\Gamma_{\eta}=d_{\eta}^{2-Q}$. That is, the functions $d_{\eta}^{2-Q}$ and $d_{\eta}^{1-Q}$ belong to $L_{l o c}^{1}\left(\mathbb{R}^{N}\right)$ and for any $\phi \in \mathscr{C}_{0}^{2}\left(\mathbb{R}^{N}\right)$ we have

$$
\int_{\mathbb{R}^{N}}(-L \phi)(\xi) \Gamma_{\eta}(\xi) d \xi=\phi(\eta) .
$$

(4) For any $i, j=1, \ldots, l$ the functions $X_{i} d_{\eta}, X_{j}\left(d_{\eta} X_{i} d_{\eta}\right)$ are bounded and $\psi_{\eta}^{2}:=\left|\nabla_{L} d_{\eta}\right|^{2} \neq 0$ almost everywhere on $\mathbb{R}^{N}$.

Finally, setting $B_{\eta}(R):=\left\{\xi \in \mathbb{R}^{N} \mid d_{\eta}(\xi)<R\right\}$, we say that $\left(\mathbf{V}_{\eta}\right)$ holds if there exist $C_{\eta}, R_{\eta}>0$ such that for any $R>R_{\eta}$, we have $\left|B_{\eta}(R)\right| \leq C_{\eta} R^{Q}$.

The conditions $\left(\mathbf{H}_{\eta}\right)$ and $\left(\mathbf{V}_{\eta}\right)$ hold if the operator $L$ is the usual Laplace operator $\Delta$ or if $L$ is a sub-laplacian on a Carnot group (see section 3 for details).

Throughout this paper we shall assume that $f: \mathbb{R}^{N} \times \mathbb{R} \rightarrow \mathbb{R}$ is a Caratheodory function.

We deal with the inequality

$$
-L u(\xi) \geq f(\xi, u(\xi)) \quad \text { a.e. on } \mathbb{R}^{N}
$$

and the equation

$$
-L u(\xi)=f(\xi, u(\xi)) \quad \text { a.e. on } \mathbb{R}^{N} .
$$

Theorem 2.2. Assume that conditions $\left(\mathbf{H}_{\eta}\right)$ and $\left(\mathbf{V}_{\eta}\right)$ hold and suppose that there exist $p>1, C_{f}>0$ such that

$$
f(\xi, t) \geq C_{f} \psi_{\eta}^{2}(\xi)|t|^{p} \quad \text { for any } t \in \mathbb{R} \text { and a.e. } \xi \in \mathbb{R}^{N} .
$$


If $u \in \mathscr{C}^{2}\left(\mathbb{R}^{N}\right)$ is a solution of (2.5), then the following inequality holds:

$$
u(\eta) \geq \int_{\mathbb{R}^{N}} \Gamma_{\eta}(\xi) f(\xi, u(\xi)) d \xi .
$$

Moreover, if $u$ is a solution of the equation (2.6), then

$$
u(\eta)=\int_{\mathbb{R}^{N}} \Gamma_{\eta}(\xi) f(\xi, u(\xi)) d \xi .
$$

Remark 2.3. From the above result it follows that if $u$ is a solution of (2.5), then either $u \equiv 0$ or $u(\eta)>0$.

Remark 2.4. We note that in (2.5) we do not assume any kind of particular behaviour on $u$ at infinity. The fact that the integral $\int_{\mathbb{R}^{N}} \Gamma_{\eta}(\xi) f(\xi, u(\xi)) d \xi$ is convergent, is a consequence of our assumptions.

Remark 2.5. The above theorem is in some sense sharp. Indeed, the negative function $u$ defined by $u(x)=u\left(x_{1}, \ldots, x_{N}\right):=-e^{x_{1}^{2}}$ satisfies

$$
-\Delta u(x)=\left(2+4 x_{1}^{2}\right) e^{x_{1}^{2}}
$$

Therefore $-\Delta u(x) \geq|u(x)|^{p}$ with $p \leq 1$.

On the other hand for $p=1$ there exist solutions that may change sign. This can be seen as follows: let $u$ be a radial solution of $-\Delta u(\xi)=|u(\xi)|$, that is, $u$ is the unique global solution of the Cauchy problem: $-u^{\prime \prime}(r)+\frac{1-N}{r} u^{\prime}(r)=|u(r)|$, $u(0)=u_{0}, u^{\prime}(0)=0$. We easily realize that if $u_{0}>0$, then this solution must change sign on $\mathbb{R}_{+}$. In fact, since $u$ is superharmonic, it follows that $u$ is decreasing. Arguing by contradiction, we assume that $u(r)>0$ for any $r \geq 0$. Thus, $u$ solves the equation

$$
-\left(r^{N-1} u^{\prime}(r)\right)^{\prime}=r^{N-1} u(r) .
$$

Integrating the last relation twice, and taking into account that $u^{\prime}(r)<0$, for every $r>0$ it follows that $u(r) \geq c_{N} r^{2} u(r)$, where $c_{N}>0$. By the strict positivity of $u$ we obtain a contradiction. Hence, there exists $r_{0}>0$ such that $u\left(r_{0}\right)=0$. This fact, with $u^{\prime}\left(r_{0}\right)<0$, implies the claim.

The following result deals with a bounded solution of (2.5) in the case $p \leq 1$.

Theorem 2.6. Assume that $\left(\mathbf{H}_{\eta}\right)$ and $\left(\mathbf{V}_{\eta}\right)$ hold. Suppose that $0 \leq p \leq 1$ and there exists $C_{f}>0$ such that

$$
f(\xi, t) \geq C_{f} \psi_{\eta}^{2}(\xi)|t|^{p} \quad \text { for any } t \in \mathbb{R} \text { and a.e. } \xi \in \mathbb{R}^{N} .
$$

Let $u \in \mathscr{C}^{2}\left(\mathbb{R}^{N}\right)$ be a bounded solution of (2.5). Then

$$
u(\eta) \geq \int_{\mathbb{R}^{N}} \Gamma_{\eta}(\xi) f(\xi, u(\xi)) d \xi
$$

holds.

Moreover, if $u$ satisfies the equation (2.6), then we have

$$
u(\eta)=\int_{\mathbb{R}^{N}} \Gamma_{\eta}(\xi) f(\xi, u(\xi)) d \xi .
$$

Therefore, if $u \not \equiv 0$ is a solution of (2.5), then $u(\eta)>0$. 
Definition 2.7. A function $u \in \mathscr{C}^{1}\left(\mathbb{R}^{N}\right)$ is a $\mathscr{C}^{1}$-weak solution of (2.5) if $f(\cdot, u(\cdot)) \in$ $L_{\text {loc }}^{1}\left(\mathbb{R}^{N}\right)$ and for any nonnegative $\varphi \in \mathscr{C}_{0}^{1}\left(\mathbb{R}^{N}\right)$ there holds

$$
\int_{\mathbb{R}^{N}}\left(\nabla_{L} u(\xi), \nabla_{L} \varphi(\xi)\right) d \xi \geq \int_{\mathbb{R}^{N}} f(\xi, u(\xi)) \varphi(\xi) d \xi .
$$

We have the following

Theorem 2.8. Assume that $\left(\mathbf{H}_{\eta}\right)$ and $\left(\mathbf{V}_{\eta}\right)$ hold. If $p \leq 1$, then the differential inequality

$$
-L u \geq C_{f} \psi_{\eta}^{2} u^{p}, \quad u>0, \quad \text { a.e. on } \mathbb{R}^{N}
$$

has no $\mathscr{C}^{1}$-weak solution.

Remark 2.9. From the proof of Theorem 2.8, it is easily seen that $L$ can be replaced by any quasilinear operator of the form $\nabla_{L}\left(A\left(\xi, u, \nabla_{L} u\right) \nabla_{L} u\right)$, where $A$ is a positive bounded function. In this case the same result holds. For distributional solutions, an analogue of the above result has been proved in [1] in the case when $\nabla_{L}$ is the usual gradient $\nabla$.

We refer the interested reader to $[3$, where the authors study existence and nonexistence of positive solutions of the equation $-\Delta u=k(x) u^{p}$ on $\mathbb{R}^{N}$; here $0<p<1$ and $k \geq 0$.

Corollary 2.10. Assume that $\left(\mathbf{H}_{\eta}\right)$ and $\left(\mathbf{V}_{\eta}\right)$ hold for any point $\eta \in \mathbb{R}^{N}$. If

$$
f(\xi, t) \geq C_{f}|t|^{p} \quad \text { for any } t \in \mathbb{R} \text { and a.e. } \xi \in \mathbb{R}^{N},
$$

with $0 \leq p \leq 1$, then (2.5) has no nontrivial bounded solution.

Remark 2.11. The statement of the above Theorem 2.6 and hence of Corollary 2.10 can be extended up to include the case $p<0$.

Assume that $\left(\mathbf{H}_{\eta}\right)$ and $\left(\mathbf{V}_{\eta}\right)$ hold. Let $u$ be a solution of (2.5) with $f(\xi, t) \geq$ $C_{f} \psi_{\eta}^{2}(\xi)|t|^{p}$ and $p>1$. If representation formula (2.8) holds, then the integral

$$
\int_{\mathbb{R}^{N}} \Gamma_{\eta}(\xi) \psi_{\eta}^{2}|u(\xi)|^{p} d \xi
$$

is convergent. Therefore

$$
\liminf _{R \rightarrow+\infty} \frac{1}{R^{2}} \int_{R \leq d_{\eta}(\xi) \leq 2 R} \psi_{\eta}^{2} \Gamma_{\eta}(\xi)|u(\xi)|^{p} d \xi=0 .
$$

Hence, by Hölder inequality, we have

$$
\liminf _{R \rightarrow+\infty} \frac{1}{R^{Q}} \int_{R \leq d_{\eta}(\xi) \leq 2 R} \psi_{\eta}^{2}|u(\xi)| d \xi=0 .
$$

The necessary condition (2.14) also turns out to be sufficient, for the validity of formula (2.8).

Indeed, we have

Theorem 2.12. Assume that $\left(\mathbf{H}_{\eta}\right)$ and $\left(\mathbf{V}_{\eta}\right)$ hold. Let $f: \mathbb{R}^{N} \times \mathbb{R} \rightarrow \mathbb{R}_{+}$be a nonnegative Caratheodory function and let $u \in \mathscr{C}^{2}\left(\mathbb{R}^{N}\right)$ be a solution of (2.5). If $u$ satisfies (2.14), then

$$
u(\eta) \geq \int_{\mathbb{R}^{N}} \Gamma_{\eta}(\xi) f(\xi, u(\xi)) d \xi
$$


Moreover, if $u$ is a solution of the equation (2.6), then

$$
u(\eta)=\int_{\mathbb{R}^{N}} \Gamma_{\eta}(\xi) f(\xi, u(\xi)) d \xi
$$

Remark 2.13. It is easy to check that if $1 \leq p<+\infty$ and $u \in L^{p}\left(\mathbb{R}^{N}\right)$, then (2.14) holds. On the other hand the reverse implication is not true. Indeed by considering

$$
u(x):=\frac{1}{1+\log (1+|x|)}, \quad x \in \mathbb{R}^{N},
$$

it follows that $u$ satisfies (2.14), but $u \notin L^{p}\left(\mathbb{R}^{N}\right)$ for any $1 \leq p<+\infty$.

The following corollary is worthwhile.

Corollary 2.14. Assume that $\left(\mathbf{H}_{\eta}\right)$ and $\left(\mathbf{V}_{\eta}\right)$ hold. Let $u \in \mathscr{C}^{2}\left(\mathbb{R}^{N}\right)$ be a solution of

$$
-L u \geq 0 \quad \text { on } \mathbb{R}^{N} .
$$

If (2.14) holds, then $u(\eta) \geq 0$. Moreover, if equality holds in (2.17), then $u(\eta)=0$.

Remark 2.15. If in the above theorem we assume that for a.e. $\xi \in \mathbb{R}^{N}$ the equation $f(\xi, t)=0$ implies $t=0$, then either $u \equiv 0$ or $u(\eta)>0, u$ being a solution of (2.5).

In what follows $\phi_{0}$ denotes a nonnegative function belonging to $\mathscr{C}_{0}^{2}(\mathbb{R})$ and such that

$$
\phi_{0}(t)=\left\{\begin{array}{ll}
1 & \text { if }|t| \leq 1, \\
0 & \text { if }|t| \geq 2,
\end{array} \quad 0 \leq \phi_{0} \leq 1\right.
$$

For any $\phi \in \mathscr{C}_{0}^{2}(\mathbb{R})$ define the function $\tilde{L}_{\eta} \phi$ by

$$
\tilde{L}_{\eta} \phi(\xi):=\phi^{\prime \prime}\left(d_{\eta}(\xi)\right)+\frac{3-Q}{d_{\eta}(\xi)} \phi^{\prime}\left(d_{\eta}(\xi)\right), \quad \xi \in \mathbb{R}^{N}, \xi \neq \eta .
$$

The following lemma plays a fundamental role for most of the results proved in this paper.

Lemma 2.16. Assume that $\left(\mathbf{H}_{\eta}\right)$ holds. Let $u \in \mathscr{C}^{2}\left(\mathbb{R}^{N}\right)$ be a solution of (2.5). Then for any nonnegative function $\phi \in \mathscr{C}_{0}^{2}(\mathbb{R})$ such that $\phi(t)=1$ for $|t| \leq 1$, we have

$$
u(\eta)-J \geq \int_{\mathbb{R}^{N}} f(\xi, u(\xi)) \Gamma_{\eta}(\xi) \phi\left(d_{\eta}(\xi)\right) d \xi,
$$

where

$$
J:=\int_{S} u(\xi) \Gamma_{\eta}(\xi) \psi_{\eta}^{2}(\xi) \tilde{L}_{\eta} \phi(\xi) d \xi
$$

and $S$ denotes the support of $\tilde{L}_{\eta} \phi$.

If $u$ also solves equation (2.6), then

$$
u(\eta)-J=\int_{\mathbb{R}^{N}} f(\xi, u(\xi)) \Gamma_{\eta}(\xi) \phi\left(d_{\eta}(\xi)\right) d \xi .
$$

Moreover, if we choose $\phi:=\phi_{0}^{\gamma}(t / R)$ with $R>1, \gamma \geq 2$ and $\phi_{0} \in \mathscr{C}_{0}^{2}(\mathbb{R})$ as in (2.18), then

$$
|J| \leq M R^{-2} \int_{S}|u(\xi)| \Gamma_{\eta}(\xi) \psi_{\eta}^{2}(\xi) \phi_{0}^{\gamma-2}\left(\frac{d_{\eta}(\xi)}{R}\right) d \xi
$$


where $S:=B_{\eta}(2 R) \backslash B_{\eta}(R)$ and

$$
M:=\left\|\frac{\tilde{L}_{\eta} \phi_{0}^{\gamma}}{\phi_{0}^{\gamma-2}}\right\|_{\infty} \gamma\left\|\phi_{0}(t) \phi_{0}^{\prime \prime}(t)+\frac{3-Q}{t} \phi_{0}(t) \phi_{0}^{\prime}(t)+(\gamma-1)\left(\phi_{0}^{\prime}(t)\right)^{2}\right\|_{\infty} .
$$

Before proving the above lemma, let us observe that assumption $\left(\mathbf{H}_{\eta}\right)$ implies the following:

$0=L\left(d_{\eta}(\xi)^{2-Q}\right)=(2-Q) d_{\eta}(\xi)^{-Q}\left((1-Q) \psi_{\eta}^{2}+d_{\eta}(\xi) L\left(d_{\eta}(\xi)\right)\right) \quad\left(\xi \in \mathbb{R}^{N} \backslash\{\eta\}\right)$. Then $L\left(d_{\eta}(\xi)\right)=(Q-1) \psi_{\eta}^{2}(\xi) / d_{\eta}(\xi)$ for any $\xi \in \mathbb{R}^{N} \backslash\{\eta\}$. Therefore, if $\phi(\xi):=$ $\varphi\left(d_{\eta}(\xi)\right)$ with $\varphi \in \mathscr{C}^{2}(\mathbb{R})$, we obtain

$$
L \phi(\xi)=\psi_{\eta}^{2}(\xi)\left(\varphi^{\prime \prime}\left(d_{\eta}(\xi)\right)+\frac{Q-1}{d_{\eta}(\xi)} \varphi^{\prime}\left(d_{\eta}(\xi)\right)\right) .
$$

In what follows we shall omit writing the dependence on $\eta$. Hence $d$ stands for $d_{\eta}, \Gamma$ for $\Gamma_{\eta}, B(R)$ for $B_{\eta}(R)$ and $\psi$ for $\psi_{\eta}$.

Proof of Lemma 2.16. First we prove (2.19). Let $m>2, \epsilon>0$ and let $\Gamma^{\epsilon}: \mathbb{R}^{N} \rightarrow$ $\mathbb{R}_{+}$be defined as follows:

$$
\Gamma^{\epsilon}(\xi):=\left(\epsilon^{m}+d^{m}(\xi)\right)^{\frac{2-Q}{m}} .
$$

From (2) and (4) of $\left(\mathbf{H}_{\eta}\right)$, we easily deduce that $\Gamma^{\epsilon} \in \mathscr{C}^{2}\left(\mathbb{R}^{N}\right)$.

Let $u$ be a solution of (2.5) and let $\tilde{\phi}:=\phi \circ d: \mathbb{R}^{N} \rightarrow \mathbb{R}_{+}$. Multiplying both sides of inequality (2.5) by $\tilde{\phi} \Gamma^{\epsilon}$ and integrating by parts, we obtain

$$
\begin{array}{rl}
\int_{\mathbb{R}^{N}} & f(\xi, u(\xi)) \Gamma^{\epsilon}(\xi) \tilde{\phi}(\xi) d \xi \leq-\int_{\mathbb{R}^{N}} u(\xi) L\left(\Gamma^{\epsilon} \tilde{\phi}\right)(\xi) d \xi=-I_{1}^{\epsilon}-I_{2}^{\epsilon} \\
=-\int_{\mathbb{R}^{N}} u(\xi) \tilde{\phi}(\xi) L \Gamma^{\epsilon}(\xi) d \xi-\int_{\mathbb{R}^{N}} u(\xi)\left[\Gamma^{\epsilon}(\xi) L \tilde{\phi}(\xi)+2\left(\nabla_{L} \tilde{\phi}, \nabla_{L} \Gamma^{\epsilon}\right)(\xi)\right] d \xi .
\end{array}
$$

Since $\Gamma^{\epsilon} \rightarrow \Gamma$ and $\nabla_{L} \Gamma^{\epsilon} \rightarrow \nabla_{L} \Gamma$ pointwise a.e. on $\mathbb{R}^{N}$, by the Lebesgue dominated convergence theorem it follows that

$$
-I_{1}^{\epsilon}=-\int_{\mathbb{R}^{N}} L(u \tilde{\phi})(\xi) \Gamma^{\epsilon}(\xi) d \xi \rightarrow-\int_{\mathbb{R}^{N}} L(u \tilde{\phi})(\xi) \Gamma(\xi) d \xi=u(\eta) \phi(0) .
$$

Next, by (2.23) and our choice on $\tilde{\phi}$, we have

$$
\begin{aligned}
I_{2}^{\epsilon} & \rightarrow \int_{\mathbb{R}^{N}} u(\xi)\left[\Gamma(\xi) L \tilde{\phi}(\xi)+2\left(\nabla_{L} \tilde{\phi}, \nabla_{L} \Gamma\right)(\xi)\right] d \xi \\
& =\int_{S} u(\xi) \Gamma(\xi) \psi^{2}(\xi)\left[\phi^{\prime \prime}(d(\xi))+\frac{3-Q}{d(\xi)} \phi^{\prime}(d(\xi))\right] d \xi \\
& =\int_{S} u(\xi) \Gamma(\xi) \psi^{2}(\xi) \tilde{L}_{\eta} \phi(\xi) d \xi=J,
\end{aligned}
$$

hence (2.19) follows.

By using the argument for proving (2.19), we easily check that identity (2.21) holds.

Finally, by choosing $\phi:=\phi_{0}^{\gamma}(t / R)$ we immediately realize that (2.22) is a consequence of the following inequalities:

$$
|J| \leq \int_{S}|u(\xi)| \Gamma(\xi) \psi^{2}\left|\tilde{L}_{\eta} \phi(\xi)\right| d \xi
$$


and

$$
\begin{aligned}
\left|\tilde{L}_{\eta} \phi(\xi)\right| & =R^{-2} \gamma\left|\phi_{0}^{\gamma-1}(t) \phi_{0}^{\prime \prime}(t)+\frac{3-Q}{t} \phi_{0}^{\gamma-1}(t) \phi_{0}^{\prime}(t)+(\gamma-1) \phi_{0}^{\gamma-2}(t)\left(\phi_{0}^{\prime}(t)\right)^{2}\right|_{\mid t=\frac{d}{R}} \\
& \leq \phi_{0}^{\gamma-2}\left(\frac{d(\xi)}{R}\right) M R^{-2} .
\end{aligned}
$$

Let us briefly comment on the role of previous lemma. Let $u \in L_{l o c}^{1}\left(\mathbb{R}^{N}\right)$ be a weak solution of (2.5) and assume that (2.7) or (2.14) is fulfilled. If (2.19) (resp. (2.21) ) is satisfied, then the representation formula (2.8) (resp. (2.9)) holds. Thus, representations formulae hold even for a weak solution $u$ of (2.5) (resp. (2.6)) provided (2.19) (resp. (2.21)) holds. For instance, this is the case when the operator $L$ is a sub-elliptic operator on a Carnot group. For sake of simplicity the next lemma deals with a special sub-elliptic operator, namely the laplacian operator $\Delta$ on $\mathbb{R}^{N}$. We refer to section 3 for the general case of sub-elliptic operators on Carnot groups.

As usual we define a weak solution of (2.5) as follows.

Definition 2.17. We say that $u$ is a weak solution of (2.5) if $u, f(\cdot, u(\cdot)) \in L_{l o c}^{1}\left(\mathbb{R}^{N}\right)$ and for any nonnegative $\varphi \in \mathscr{C}_{0}^{2}\left(\mathbb{R}^{N}\right)$ we have

$$
-\int_{\mathbb{R}^{N}} u(\xi) L \varphi(\xi) d \xi \geq \int_{\mathbb{R}^{N}} f(\xi, u(\xi)) \varphi(\xi) d \xi .
$$

In a similar way we define a weak solution of (2.6).

Lemma 2.18. Let $u$ be a weak solution of

$$
-\Delta u(\xi) \geq f(\xi, u(\xi)) \quad \text { a.e. on } \mathbb{R}^{N}
$$

and let $\eta$ be a Lebesgue point of $u$. Then (2.19) holds for any nonnegative function $\phi \in \mathscr{C}_{0}^{2}(\mathbb{R})$ such that $\phi(t)=1$ for $|t| \leq 1$.

In particular if $u$ is a solution of

$$
-\Delta u(\xi)=f(\xi, u(\xi)) \text { a.e. on } \mathbb{R}^{N},
$$

then (2.21) holds.

Proof of Lemma 2.18. It is well known that the fundamental solution of $-\Delta$ at $\eta$ is given by $\Gamma_{\eta}(\xi)=\left(c_{E}|\eta-\xi|\right)^{2-N}$, where $c_{E}$ is a suitable normalization constant. Therefore the function $d_{\eta}$ is given by $d_{\eta}(\xi)=d(\xi)=c_{E}|\eta-\xi|$ and $\psi_{\eta}=1$. Let $\phi \in \mathscr{C}_{0}^{2}(\mathbb{R})$ be such that $\phi(t)=1$ for $|t| \leq 1$ and set $\tilde{\phi}:=\phi \circ d: \mathbb{R}^{N} \rightarrow \mathbb{R}_{+}$, that is, $\tilde{\phi}=\phi\left(c_{E}|\eta-\cdot|\right)$. Next we choose the test function $\varphi$ in the definition of a weak solution of (2.25), as $\varphi:=\tilde{\phi} \Gamma^{\epsilon}$. Our principal claim is to show that

$$
-I_{1}^{\epsilon}=-\int_{\mathbb{R}^{N}} u(\xi) \tilde{\phi}(\xi) L \Gamma^{\epsilon}(\xi) d \xi \rightarrow u(\eta) \quad \text { as } \quad \epsilon \rightarrow 0 .
$$

If $u \in \mathscr{C}^{2}\left(\mathbb{R}^{N}\right)$, then arguing as in (2.24) and using the Lebesgue dominated convergence theorem, (2.27) follows.

Now we consider the general case $u \in L_{l o c}^{1}\left(\mathbb{R}^{N}\right)$. A simple computation yields

$$
-I_{1}^{\epsilon}=(N-2)(N-2+m) \int_{\mathbb{R}^{N}}(u \tilde{\phi})(\xi) \frac{\epsilon^{m} d_{\eta}^{m-2}(\xi)}{\left(\epsilon^{m}+d_{\eta}^{m}(\xi)\right)^{\frac{N-2}{m}+2}} d \xi .
$$


Let $g_{1}: \mathbb{R}^{N} \rightarrow \mathbb{R}$ be defined by

$$
g_{1}(\xi):=\frac{c_{E}|\xi|^{m-2}}{\left(1+c_{E}^{m}|\xi|^{m}\right)^{\frac{N-2}{m}+2}} \quad \text { for } \xi \in \mathbb{R}^{N},
$$

and set $g_{\epsilon}(\xi):=\frac{1}{\epsilon^{N}} g_{1}\left(\frac{\xi}{\epsilon}\right)$. Clearly we have $\int g_{\epsilon}=\int g_{1}<+\infty$. Now we rewrite $-I_{1}^{\epsilon}$ as follows:

$$
-I_{1}^{\epsilon}=(N-2)(N-2+m)\left(u \tilde{\phi} * g_{\epsilon}\right)(\eta) .
$$

If $\tau$ is a Lebesgue point of $u \tilde{\phi}$, it follows that $\left(u \tilde{\phi} * g_{\epsilon}\right)(\tau) \rightarrow(u \tilde{\phi})(\tau) \int g_{1}$. On the other hand, if $\eta$ is a Lebesgue point of $u$, and hence of $u \tilde{\phi}$, we obtain

$$
-I_{1}^{\epsilon} \rightarrow u(\eta)(N-2)(N-2+m) \int_{\mathbb{R}^{N}} g_{1}(\xi) d \xi=: u(\eta) C_{1} .
$$

Since $C_{1}$ does not depend on $u$ and (2.27) holds when $u$ is smooth, we conclude that $C_{1}=1$. This prove the claim.

Proof of Theorem 2.2. Let $u$ be a solution of (2.5) and choose $\phi(t)=\phi_{R}(t):=$ $\phi_{0}^{\gamma}(t / R)$ with $R>1, \gamma>2$ and $\phi_{0} \in \mathscr{C}_{0}^{2}(\mathbb{R})$ as in (2.18). From Lemma [2.16] it follows that $u$ and $J$ satisfy (2.19) and (2.22), respectively. An application of Young inequality to (2.22) gives

$$
\begin{aligned}
|J| & \leq M \int_{S}|u(\xi)| \Gamma(\xi)^{\frac{1}{p}} \psi^{\frac{2}{p}}(\xi) \phi_{0}^{\gamma-2}(d(\xi) / R) \Gamma(\xi)^{\frac{1}{p^{\prime}}} \psi^{\frac{2}{p^{\prime}}}(\xi) R^{-2} d \xi \\
& \leq \frac{M \delta^{p}}{p} \int_{S}|u(\xi)|^{p} \Gamma(\xi) \psi^{2}(\xi) \phi_{0}^{p(\gamma-2)}(d(\xi) / R) d \xi+\frac{M}{p^{\prime} \delta^{p^{\prime}}} R^{-2 p^{\prime}} \int_{S} \Gamma(\xi) \psi^{2}(\xi) d \xi .
\end{aligned}
$$

Next we choose $\gamma \geq 2+\frac{2}{p-1}$ so that $p(\gamma-2) \geq \gamma$. Thus from (2.7), $\left(\mathbf{H}_{\eta}\right)$ and $\left(\mathbf{V}_{\eta}\right)$, we deduce that

$$
\begin{aligned}
|J| & \leq \frac{M \delta^{p}}{p} \int_{S}|u(\xi)|^{p} \Gamma(\xi) \psi^{2}(\xi) \phi_{0}^{p \gamma}(d(\xi) / R) d \xi \\
& +\frac{M\left\|\psi^{2}\right\|_{\infty}}{p^{\prime} \delta^{p^{\prime}}} R^{-2 p^{\prime}} \int_{S} d^{2-Q}(\xi) d \xi \\
& \leq \frac{M \delta^{p}}{p C_{f}} \int_{S} f(\xi, u(\xi)) \Gamma(\xi) \phi_{R}(d(\xi)) d \xi+\frac{M\left\|\psi^{2}\right\|_{\infty} C_{\eta}}{p^{\prime} \delta^{p^{\prime}}} R^{2-2 p^{\prime}}
\end{aligned}
$$

for $R>R_{\eta}$. Choosing $\delta$ sufficiently small, from (2.19) it follows that

$$
\left(1-C_{1} \delta^{p}\right) \int_{\mathbb{R}^{N}} f(\xi, u(\xi)) \Gamma_{\eta}(\xi) \phi_{R}(d(\xi)) d \xi \leq u(\eta)+\frac{C_{2}}{\delta^{p^{\prime}}} R^{2-2 p^{\prime}} .
$$

Next, by letting $R \rightarrow+\infty$ and then $\delta \rightarrow 0$ in the above inequality, we deduce that (2.8) holds.

Now let $u$ be a solution of (2.6). From (2.8) and Lemma 2.16 we have

$$
\int_{\mathbb{R}^{N}} f(\xi, u(\xi)) \Gamma_{\eta}(\xi) \phi_{R}(\xi) d \xi=u(\eta)-J
$$

Inequality (2.30) together with (2.7) yields

$$
\left(1+C_{1} \delta^{p}\right) \int_{\mathbb{R}^{N}} f(\xi, u(\xi)) \Gamma_{\eta}(\xi) \phi_{R}(\xi) d \xi \geq u(\eta)-\frac{C_{2}}{\delta^{p^{\prime}}} R^{2-2 p^{\prime}},
$$

and by letting $R \rightarrow+\infty$, it follows that

$$
\left(1+C_{1} \delta^{p}\right) \int_{\mathbb{R}^{N}} f(\xi, u(\xi)) \Gamma_{\eta}(\xi) d \xi \geq u(\eta) .
$$


Notice that by (2.8) the integral in left-hand side of (2.31) is convergent. Taking the limit as $\delta \rightarrow 0$ we complete the proof.

Proof of Theorem 2.6. Let $u \in \mathscr{C}^{2}\left(\mathbb{R}^{N}\right)$ be a nontrivial bounded solution of (2.5). Let $\phi_{R}(d(\xi)):=\phi_{0}^{2}(d(\xi) / R)$, where $R>1$. Here, $\phi_{0} \in \mathscr{C}_{0}^{2}(\mathbb{R})$ is chosen as in (2.18) and $J$ is defined by (2.20).

From Lemma 2.16 it follows that $u$ and $J$ satisfy (2.19) and (2.22), respectively. Moreover, if $u$ solves (2.6), then (2.21) holds.

We claim that $J \rightarrow 0$ as $R \rightarrow+\infty$. Indeed, since $u$ is bounded, for $R>R_{\eta}$, we obtain

$$
|J| \leq\|u\|_{\infty}\left\|\psi^{2}\right\|_{\infty} M R^{-2} \int_{S} d^{2-Q}(\xi) d \xi \leq\|u\|_{\infty}\left\|\psi^{2}\right\|_{\infty} M C_{\eta} .
$$

Therefore inequality (2.19) yields

$$
\int_{\mathbb{R}^{N}} f(\xi, u(\xi)) \Gamma_{\eta}(\xi) \phi_{R}(d(\xi)) d \xi \leq u(\eta)+\|u\|_{\infty}\left\|\psi^{2}\right\|_{\infty} M C_{\eta} .
$$

Noticing that in the above estimate the right-hand side does not depend on $R$ and $f$ is nonnegative, we deduce that

$$
C_{1}:=\int_{\mathbb{R}^{N}} f(\xi, u(\xi)) \Gamma_{\eta}(\xi) d \xi<+\infty
$$

Since $p \leq 1$ and $0 \neq v \in L^{\infty}\left(\mathbb{R}^{N}\right)$, we have

$$
|v(\xi)| \leq|v(\xi)|^{p}\|v\|_{\infty}^{1-p} \quad \text { a.e. } \xi \in \mathbb{R}^{N} .
$$

Thus, from (2.22) we get

$$
\begin{aligned}
|J| & \leq \frac{M}{R^{2}} \int_{S}|u(\xi)| \Gamma(\xi) \psi^{2}(\xi) d \xi \leq \frac{M}{R^{2}}\|u\|_{\infty}^{1-p} \int_{S}|u(\xi)|^{p} \Gamma(\xi) \psi^{2}(\xi) d \xi \\
& \leq \frac{M}{C_{f} R^{2}}\|u\|_{\infty}^{1-p} \int_{S} f(\xi, u(\xi)) \Gamma_{\eta}(\xi) d \xi \leq \frac{M}{C_{f} R^{2}}\|u\|_{\infty}^{1-p} C_{1}=: \frac{C}{R^{2}} .
\end{aligned}
$$

This proves the claim and consequently the validity of (2.8).

Now suppose that $u$ is a solution of equation (2.6). From (2.21) it follows that

$$
\int_{B(2 R)} f(\xi, u(\xi)) \Gamma_{\eta}(\xi) d \xi \geq u(\eta)-\frac{C}{R^{2}} .
$$

By taking the limit as $R \rightarrow+\infty$ we conclude the proof.

Remark 2.19. The fact that $\int_{\mathbb{R}^{N}} f(\xi, u(\xi)) \Gamma_{\eta}(\xi) d \xi<+\infty$ is a consequence of only two assumptions: the boundedness of $u$ and the positivity of $f$.

Proof of Theorem 2.12, Let $u$ be a solution of (2.5) and hence of (2.19). Let $\phi_{0} \in$ $\mathscr{C}^{2}\left(\mathbb{R}^{N}\right)$ be as in (2.18) and set $\phi(t)=\phi_{R}(t):=\phi_{0}^{2}(t / R)$ with $R>1$. From (2.19) and the positivity of $f$ we deduce

$$
\int_{B(R)} f(\xi, u(\xi)) \Gamma_{\eta}(\xi) d \xi \leq \int_{\mathbb{R}^{N}} f(\xi, u(\xi)) \Gamma_{\eta}(\xi) \phi_{R}(d(\xi)) d \xi \leq u(\eta)+|J| .
$$

We claim that $J \rightarrow 0$ as $R \rightarrow+\infty$. Indeed, from (2.22) we obtain

$$
|J| \leq \frac{M}{R^{2}} \int_{S}|u(\xi)| d^{2-Q} \psi^{2}(\xi) d \xi \leq \frac{M}{R^{Q}} \int_{B(2 R) \backslash B(R)}|u(\xi)| \psi^{2}(\xi) d \xi .
$$

Hence, by (2.14) the claim follows and consequently inequality (2.8) holds. 
Next, suppose that $u$ solves equation (2.6). Then

$$
u(\eta) \geq \int_{B(2 R)} f(\xi, u(\xi)) \Gamma_{\eta}(\xi) d \xi \geq \int_{\mathbb{R}^{N}} f(\xi, u(\xi)) \Gamma_{\eta}(\xi) \phi_{R}(d(\xi)) d \xi \geq u(\eta)-|J| .
$$

By letting $R \rightarrow+\infty$ in the above inequalities, we easily deduce that (2.9) holds.

Proof of Theorem 2.8. Let $u>0$ be a $\mathscr{C}^{1}$-weak solution of (2.11). The function $\varphi:=\phi u^{\alpha}$ is an admissible test function for every $\phi \in \mathscr{C}_{0}^{1}\left(\mathbb{R}^{N}\right)$ and $\alpha<0$, hence

$$
\begin{gathered}
\int_{\mathbb{R}^{N}} C_{f} \psi^{2}(\xi) u^{p+\alpha}(\xi) \phi(\xi) d \xi \leq \alpha \int_{\mathbb{R}^{N}}\left|\nabla_{L} u\right|^{2}(\xi) u^{\alpha-1}(\xi) \phi(\xi) d \xi \\
+\int_{\mathbb{R}^{N}}\left(\nabla_{L} u, \nabla_{L} \phi\right)(\xi) u^{\alpha}(\xi) d \xi \\
\leq \alpha \int_{\mathbb{R}^{N}}\left|\nabla_{L} u\right|^{2}(\xi) u^{\alpha-1}(\xi) \phi(\xi) d \xi+\int_{\mathbb{R}^{N}}\left|\nabla_{L} u\right|(\xi)\left|\nabla_{L} \phi\right|(\xi) u^{\alpha}(\xi) d \xi .
\end{gathered}
$$

Applying Hölder and Young inequalities to the second integral on right-hand side of the above inequalities, we deduce

$$
\begin{aligned}
\int_{\mathbb{R}^{N}} C_{f} \psi^{2}(\xi) u^{p+\alpha}(\xi) \phi(\xi) d \xi \leq\left(\alpha+\frac{1}{2}\right) & \int_{\mathbb{R}^{N}}\left|\nabla_{L} u\right|^{2}(\xi) u^{\alpha-1}(\xi) \phi(\xi) d \xi \\
& +\frac{1}{2} \int_{\mathbb{R}^{N}} \frac{\left|\nabla_{L} \phi\right|^{2}(\xi)}{\phi(\xi)} u^{\alpha+1}(\xi) d \xi
\end{aligned}
$$

First we consider the case $p<1$. By choosing $\phi=\tilde{\phi}\left(d_{\eta}(\cdot)\right)$ with $\tilde{\phi} \in \mathscr{C}_{0}^{1}(\mathbb{R})$ as in (2.18), it follows that

$$
\left|\nabla_{L} \phi\right|^{2}(\xi)=\psi^{2}(\xi)\left|\tilde{\phi}^{\prime}\right|(d(\xi))
$$

Setting $\beta:=\frac{p+\alpha}{\alpha+1}$ with $\alpha<-1$, it results in the fact that $\beta>1$. Again applying Young inequalities with parameter $\delta>0$ and exponent $\beta$ to the last term of the right-hand side of (2.33), we arrive at

$$
\begin{gathered}
\int_{\mathbb{R}^{N}} C_{f} \psi^{2}(\xi) u^{p+\alpha}(\xi) \phi(\xi) d \xi+\left(|\alpha|-\frac{1}{2}\right) \int_{\mathbb{R}^{N}}\left|\nabla_{L} u\right|^{2}(\xi) u^{\alpha-1}(\xi) \phi(\xi) d \xi \\
\quad \leq \frac{\delta^{\beta}}{2 \beta} \int_{\mathbb{R}^{N}} \psi^{2}(\xi) u^{p+\alpha}(\xi) \phi(\xi) d \xi+\frac{1}{2 \delta^{\beta^{\prime} \beta^{\prime}}} \int_{\mathbb{R}^{N}} \psi^{2}(\xi) \frac{\left|\tilde{\phi}^{\prime}\right|^{2 \beta^{\prime}}}{\tilde{\phi}^{2 \beta^{\prime}-1}}(d(\xi)) d \xi
\end{gathered}
$$

Next we choose $\phi=\tilde{\phi} \circ d$, where $\tilde{\phi}(t):=\phi_{0}^{\gamma}(t / R)$ and $\phi_{0} \in \mathscr{C}_{0}^{1}(\mathbb{R})$ is as in (2.18) with $\gamma \geq 2 \beta^{\prime}$. With this choice, the last integral in (2.34) is finite and

$$
\left(C_{f}-\frac{\delta^{\beta}}{2 \beta}\right) \int_{B(R)} \psi^{2}(\xi) u^{p+\alpha}(\xi) d \xi \leq \frac{M}{2 \delta^{\beta^{\prime}} \beta^{\prime} R^{2 \beta^{\prime}}} \int_{B(2 R) \backslash B(R)} \psi^{2}(\xi) d \xi \leq C_{1} R^{Q-2 \beta^{\prime}}
$$

for any $R>R_{\eta}$. Finally, by selecting $\alpha<\min \{-1,((Q-2) p-Q) / 2\}$, it follows that $Q-2 \beta^{\prime}<0$. Thus from (2.35), and $\delta$ sufficiently small, we deduce that $\int_{\mathbb{R}^{N}} \psi^{2} u^{p+\alpha}=0$ which implies $u \equiv 0$. This contradiction completes the proof in the case $p<1$.

Next we consider the case $p=1$. By choosing $\alpha=-1$ and $\phi(\xi)=\phi_{0}^{2}(d(\xi) / R)$ as above, from (2.33), we obtain

$$
C_{f} \int_{B(R)} \psi^{2}(\xi) d \xi+\frac{1}{2} \int_{B(R)} \frac{\left|\nabla_{L} u\right|^{2}}{u^{2}}(\xi) d \xi \leq \frac{\left\|4 \phi_{0}^{\prime}\right\|_{\infty}}{2 R^{2}} \int_{B(2 R) \backslash B(R)} \psi^{2}(\xi) d \xi
$$


hence

$$
f_{1}(R) \leq C_{1} \frac{f_{1}(2 R)}{R^{2}}
$$

where $f_{1}(R):=\int_{B(R)} \psi^{2} d \xi$. By our assumption on $\psi$, it follows that $f_{1}$ is a positive increasing function. Let $l:=\lim _{R \rightarrow+\infty} f_{1}(R)$. From (2.37) we deduce that $l=+\infty$ and, by letting $R \rightarrow+\infty$ in (2.37), we conclude that $f_{2}(R):=\frac{f_{1}(R)}{R^{2}} \rightarrow+\infty$ as $R \rightarrow+\infty$. Next, by substituting $f_{1}(R)=R^{2} f_{2}(R)$ in (2.37), we obtain

$$
f_{2}(R) \leq C_{2} \frac{f_{2}(2 R)}{R^{2}}
$$

with $C_{2}=4 C_{1}$. Iterating this procedure, it follows that for any $n>1$ there exists $f_{n}(R)$ such that $f_{n-1}=R^{2} f_{n}(R), f_{n}(R) \leq R^{-2} f_{n}(2 R)$ and $f_{n}(R) \rightarrow+\infty$ as $R \rightarrow+\infty$. Therefore,

$$
R^{2 n} f_{n+1}(R)=f_{1}(R)=\int_{B(R)} \psi^{2}(\xi) d \xi \leq\left\|\psi^{2}\right\|_{\infty} C_{\eta} R^{Q}, \quad R>R_{\eta} .
$$

By choosing $n$ sufficiently large we reach a contradiction, thereby concluding the proof.

The above results can be generalized for inequalities of the type

$$
-L(a u)(\xi) \geq f(\xi, u(\xi)) \quad \text { a.e. on } \mathbb{R}^{N}
$$

or for equations of the form

$$
-L(a u)(\xi)=f(\xi, u(\xi)) \quad \text { a.e. on } \mathbb{R}^{N} .
$$

Here $a$ and $f$ are given functions with a certain behaviour at infinity. Here, we deal only with a simple result in this direction.

Let $f(\xi, t) \geq C_{f} \psi_{\eta}^{2}(\xi)|t|^{p}$ with $p>1$ and let $a$ be a bounded function.

The following result can be proved by a slight modification of the proof of Theorem 2.2 .

Theorem 2.20. Assume that $\left(\mathbf{H}_{\eta}\right)$ and $\left(\mathbf{V}_{\eta}\right)$ hold. Let $a \in \mathscr{C}^{2}\left(\mathbb{R}^{N}\right) \cap L^{\infty}\left(\mathbb{R}^{N}\right)$, $p>1$ and $f(\xi, t) \geq C_{f} \psi_{\eta}^{2}(\xi)|t|^{p}$. If $u$ is a solution of (2.39), then we have

$$
a(\eta) u(\eta) \geq \int_{\mathbb{R}^{N}} \Gamma_{\eta}(\xi) f(\xi, u(\xi)) d \xi .
$$

Moreover, if $u$ solves (2.40), then

$$
a(\eta) u(\eta)=\int_{\mathbb{R}^{N}} \Gamma_{\eta}(\xi) f(\xi, u(\xi)) d \xi .
$$

Remark 2.21. If $u$ is a solution of

$$
-\Delta(a u)(\xi) \geq f(\xi, u(\xi)) \text { a.e. on } \mathbb{R}^{N},
$$

and the function $a$ vanishes at some point, then $u \equiv 0$ on $\mathbb{R}^{N}$.

An application of the above result gives the following:

i) Let $u \in \mathscr{C}^{3}\left(\mathbb{R}^{N}\right)$ be a globally Lipschitz nontrivial solution of

$$
-\Delta\left(|\nabla u|^{2} u\right) \geq|u|^{p} \quad \text { on } \mathbb{R}^{N}, p>1 \text {. }
$$

Then $u$ is strictly positive and without critical points. Moreover, the integral in the right-hand side of 2.41) is convergent and consequently $u$ cannot be a ground state. 
ii) Let $u \in \mathscr{C}^{2}\left(\mathbb{R}^{N}\right)$ be a bounded nontrivial solution of the inequality

$$
-\Delta\left(|u|^{m-1} u\right) \geq C_{f}|u|^{p}, \quad u \not \equiv 0, \quad \text { a.e. on } \mathbb{R}^{N}, p>1, m>1 \text {. }
$$

Applying (2.41) with the choice $a(\xi)=|u(\xi)|^{m-1}$, it follows that $u>0$ on $\mathbb{R}^{N}$. Thus, $v:=u^{m}$ satisfies the inequality

$$
-\Delta v \geq C_{f} v^{p / m}, \quad v>0, \quad \text { on } \mathbb{R}^{N},
$$

which has a bounded solutions if and only if $p / m>N /(N-2)$. So, 2.43) has a bounded solution if and only if $p / m>N /(N-2)$. Moreover nontrivial solutions are strictly positive.

2.1. System of inequalities. In this section we shall briefly indicate how some of the preceding results can be generalized to a class of systems of semilinear equations and inequalities on $\mathbb{R}^{N}$.

We have the following.

Theorem 2.22. Suppose that the hypotheses $\left(\mathbf{H}_{\eta}\right)$ and $\left(\mathbf{V}_{\eta}\right)$ are satisfied. Let $u, v \in \mathscr{C}^{2}\left(\mathbb{R}^{N}\right)$ be a solution of

$$
\begin{cases}-L u \geq f(\xi, u, v) & \text { a.e. on } \mathbb{R}^{N}, \\ -L v \geq g(\xi, u, v) & \text { a.e. on } \mathbb{R}^{N},\end{cases}
$$

where $f, g: \mathbb{R}^{N} \times \mathbb{R} \times \mathbb{R} \rightarrow \mathbb{R}$ are Caratheodory functions such that there exist $p, q>1, C_{f}, C_{g}>0$ such that for a.e. $\xi \in \mathbb{R}^{N}$ and any $t, s \in \mathbb{R}$, there holds

$$
f(\xi, t, s) \geq C_{f} \psi_{\eta}^{2}|s|^{p}, \quad g(\xi, t, s) \geq C_{g} \psi_{\eta}^{2}|t|^{q} .
$$

Then

$$
u(\eta) \geq \int_{\mathbb{R}^{N}} f(\xi, u(\xi), v(\xi)) \Gamma_{\eta}(\xi) d \xi, \quad v(\eta) \geq \int_{\mathbb{R}^{N}} g(\xi, u(\xi), v(\xi)) \Gamma_{\eta}(\xi) d \xi
$$

Therefore, either $u=0$ and $v=0$, or $u(\eta)>0$ and $v(\eta)>0$.

Moreover, if equalities hold in (2.44), then

$$
u(\eta)=\int_{\mathbb{R}^{N}} f(\xi, u(\xi), v(\xi)) \Gamma_{\eta}(\xi) d \xi, \quad v(\eta)=\int_{\mathbb{R}^{N}} g(\xi, u(\xi), v(\xi)) \Gamma_{\eta}(\xi) d \xi .
$$

Let $(u, v)$ be a solution of (2.44). Then for any positive functions $\phi_{i} \in \mathscr{C}_{0}^{2}\left(\mathbb{R}^{N}\right)$, $i=1,2$, we have

$$
\left\{\begin{array}{l}
-\int_{\mathbb{R}^{N}} u(\xi) L \phi_{1}(\xi) d \xi \geq \int_{\mathbb{R}^{N}} f(\xi, u, v) \phi_{1}(\xi) d \xi, \\
-\int_{\mathbb{R}^{N}} v(\xi) L \phi_{2}(\xi) d \xi \geq \int_{\mathbb{R}^{N}} g(\xi, u, v) \phi_{2}(\xi) d \xi .
\end{array}\right.
$$

Arguing as in Lemma 2.16 we prove

Lemma 2.23. Let the hypotheses $\left(\mathbf{H}_{\eta}\right)$ be fulfilled. Let $(u, v)$ be a solution of (2.44). For any positive functions $\phi_{i} \in \mathscr{C}_{0}^{2}(\mathbb{R})$ such that $\phi_{i}(t)=1$ for $|t| \leq 1$ $(i=1,2)$, we have

$$
\begin{aligned}
& u(\eta)-I_{2}^{\prime} \geq \int_{\mathbb{R}^{N}} f(\xi, u(\xi), v(\xi)) \Gamma_{\eta}(\xi) \phi_{1}\left(d_{\eta}(\xi)\right) d \xi \\
& v(\eta)-I_{2}^{\prime \prime} \geq \int_{\mathbb{R}^{N}} g(\xi, u(\xi), v(\xi)) \Gamma_{\eta}(\xi) \phi_{2}\left(d_{\eta}(\xi)\right) d \xi
\end{aligned}
$$


where

$$
\begin{aligned}
I_{2}^{\prime} & :=\int_{S_{1}} u(\xi) \Gamma_{\eta}(\xi) \psi_{\eta}^{2}(\xi)\left[\phi_{1}^{\prime \prime}\left(d_{\eta}(\xi)\right)+\frac{3-Q}{d_{\eta}(\xi)} \phi_{1}^{\prime}\left(d_{\eta}(\xi)\right)\right] d \xi, \\
I_{2}^{\prime \prime} & :=\int_{S_{2}} v(\xi) \Gamma_{\eta}(\xi) \psi_{\eta}^{2}(\xi)\left[\phi_{2}^{\prime \prime}\left(d_{\eta}(\xi)\right)+\frac{3-Q}{d_{\eta}(\xi)} \phi_{2}^{\prime}\left(d_{\eta}(\xi)\right)\right] d \xi,
\end{aligned}
$$

and $S_{i}$ is the support of $\phi_{i}^{\prime \prime}\left(d_{\eta}(\xi)\right)+\frac{3-Q}{d_{\eta}(\xi)} \phi_{i}^{\prime}\left(d_{\eta}(\xi)\right)$.

If $(u, v)$ is a solution of

$$
\begin{aligned}
& -L u=f(\xi, u, v) \quad \text { a.e. on } \mathbb{R}^{N}, \\
& -L v=g(\xi, u, v) \quad \text { a.e. on } \mathbb{R}^{N},
\end{aligned}
$$

then equality holds in (2.48).

Proof. We choose $\phi_{1}(\xi)=\phi_{2}(\xi)=\phi_{R}(\xi):=\phi_{0}^{\gamma}\left(\frac{d(\xi)}{R}\right)$ with $R>1, \phi_{0} \in \mathscr{C}_{0}^{2}(\mathbb{R})$ as in (2.18) and $\gamma$ large enough. Proceeding as in the scalar case, using Hölder and Young inequalities, we obtain for $\delta>0$

$$
\begin{aligned}
\left|I_{2}^{\prime}\right| & \leq \frac{\delta^{q}}{q} \int_{\mathbb{R}^{N}}|u(\xi)|^{q} \Gamma_{\eta}(\xi) \psi_{\eta}^{2}(\xi) \phi_{R}(\xi) d \xi+\frac{C}{q^{\prime} \delta^{q^{\prime}}} R^{2-2 q^{\prime}} \\
& \leq \frac{\delta^{q}}{q C_{g}} \int_{\mathbb{R}^{N}} g(\xi, u, v) \Gamma_{\eta}(\xi) \phi_{R}(\xi) d \xi+\frac{C}{q^{\prime} \delta^{q^{\prime}}} R^{2-2 q^{\prime}}
\end{aligned}
$$

and analogously for $\epsilon>0$

$$
\left|I_{2}^{\prime \prime}\right| \leq \frac{\epsilon^{p}}{p C_{f}} \int_{\mathbb{R}^{N}} f(\xi, u, v) \Gamma_{\eta}(\xi) \phi_{R}(\xi) d \xi+\frac{C}{p^{\prime} \epsilon^{p^{\prime}}} R^{2-2 p^{\prime}}
$$

Hence for $R>R_{\eta}$ we obtain

$$
\begin{aligned}
\int_{\mathbb{R}^{N}} f(\xi, u(\xi), v(\xi)) \Gamma_{\eta}(\xi) \phi_{R}(\xi) d \xi & \\
& \leq u(\eta)+\frac{\delta^{q}}{q C_{g}} \int_{\mathbb{R}^{N}} g(\xi, u, v) \Gamma_{\eta}(\xi) \phi_{R}(\xi) d \xi+\frac{C}{q^{\prime} \delta^{q^{\prime}}} R^{2-2 q^{\prime}}
\end{aligned}
$$

and

$$
\begin{aligned}
& \int_{\mathbb{R}^{N}} g(\xi, u(\xi), v(\xi)) \Gamma_{\eta}(\xi) \phi_{R}(\xi) d \xi \\
& \quad \leq v(\eta)+\frac{\epsilon^{p}}{p C_{f}} \int_{\mathbb{R}^{N}} f(\xi, u, v) \Gamma_{\eta}(\xi) \phi_{R}(\xi) d \xi+\frac{C}{p^{\prime} \epsilon^{p^{\prime}}} R^{2-2 p^{\prime}}
\end{aligned}
$$

The last inequalities imply

$$
\begin{array}{rl}
\left(1-\frac{\delta^{q} \epsilon^{p}}{q p C_{f} C_{g}}\right) \int_{\mathbb{R}^{N}} & f(\xi, u(\xi), v(\xi)) \Gamma_{\eta}(\xi) \phi_{R}(\xi) d \xi \\
\leq & u(\eta)+\frac{\delta^{q}}{q C_{g}} v(\eta)+\frac{C \delta^{q}}{q C_{g} \epsilon^{p^{\prime}}} R^{2-2 p^{\prime}}+\frac{C}{\delta^{q^{\prime}}} R^{2-2 q^{\prime}}
\end{array}
$$

Letting $R \rightarrow+\infty$ and then $\delta \rightarrow 0$ we obtain the first part of (2.45). The other is analogue. The proof of the last part of the theorem follows as in the scalar case. 


\section{Carnot Group}

In this section we shall study some of the preceding results in the framework of Carnot groups.

We begin by quoting some preliminary facts concerning Carnot groups (for more information and proofs we refer the interested reader to [5, 6]; see also the survey 10]). A Carnot group is a connected, simply connected, nilpotent Lie group $\mathbb{G}$ of dimension $N \geq 2$ with graded Lie algebra $\mathcal{G}=V_{1} \oplus \cdots \oplus V_{r}$ such that $\left[V_{1}, V_{i}\right]=V_{i+1}$ for $i=1, \ldots, r-1$ and $\left[V_{1}, V_{r}\right]=0$. A Carnot group $\mathbb{G}$ of dimension $N$ can be identified, up to an isomorphism, with the structure of homogeneous Carnot group $\left(\mathbb{R}^{N}, \circ, \delta_{\lambda}\right)$ defined as follows. We identify $\mathbb{G}$ with $\mathbb{R}^{N}$ endowed with a Lie group law $\circ$. We consider $\mathbb{R}^{N}$ split in $r$ subspaces $\mathbb{R}^{N}=\mathbb{R}^{n_{1}} \times \mathbb{R}^{n_{2}} \times \cdots \times \mathbb{R}^{n_{r}}$ with $n_{1}+n_{2}+\cdots+n_{r}=N$ and $\xi=\left(\xi^{(1)}, \ldots, \xi^{(r)}\right)$ with $\xi^{(i)} \in \mathbb{R}^{n_{i}}$. We shall assume that there exists a family of Lie group automorphisms, called a dilation, $\delta_{\lambda}$ with $\lambda>0$ of the form

$$
\delta_{\lambda}(\xi)=\left(\lambda \xi^{(1)}, \lambda^{2} \xi^{(2)}, \ldots, \lambda^{r} \xi^{(r)}\right) .
$$

The Lie algebra of left-invariant vector fields on $\left(\mathbb{R}^{N}, \circ\right)$ is $\mathcal{G}$. For $i=1, \ldots, n_{1}=l$ let $X_{i}$ be the unique vector field in $\mathcal{G}$ that coincides with $\partial / \partial \xi_{i}^{(1)}$ at the origin. We require that the Lie algebra generated by $X_{1}, \ldots, X_{n_{1}}$ is the whole $\mathcal{G}$.

With the above hypotheses, we call $\mathbb{G}=\left(\mathbb{R}^{N}, \circ, \delta_{\lambda}\right)$ a homogeneous Carnot group. The canonical sub-laplacian on $\mathbb{G}$ is the second order differential operator $\Delta_{G}=\sum_{i=1}^{l} X_{i}^{2}$. Let $Y_{1}, \ldots, Y_{l}$ be a basis of $\operatorname{span}\left\{X_{1}, \ldots, X_{l}\right\}$; the second order differential operator $L=\sum_{i=1}^{l} Y_{i}^{2}$ is called a sub-laplacian on $\mathbb{G}$. We denote by $Q=\sum_{i=1}^{r} i n_{i}$ the homogeneous dimension of $\mathbb{G}$. In the sequel we assume $Q \geq 3$.

We shall list some properties and known results about homogeneous Carnot groups.

The Lebesgue measure is the bi-invariant Haar measure. For any measurable set $E \subset \mathbb{R}^{N}$, we have $\left|\delta_{\lambda}(E)\right|=\lambda^{Q}|E|$. Since $Y_{1}, \ldots, Y_{l}$ generate the whole $\mathcal{G}$, any sub-Laplacian $L$ satisfies the Hörmander's hypoellipticity condition. Moreover, the vector fields $Y_{1}, \ldots, Y_{l}$ are homogeneous of degree 1 with respect to $\delta_{\lambda}$.

A continuous function $d: \mathbb{R}^{N} \rightarrow \mathbb{R}_{+}$is called a homogeneous norm on $\mathbb{G}$ if it is smooth on $\mathbb{R}^{N} \backslash\{0\}, d\left(\xi^{-1}\right)=d(\xi), d(\xi)=0$ if and only if $\xi=0$ and it is homogeneous of degree 1 with respect to $\delta_{\lambda}$ (i.e. $d\left(\delta_{\lambda}(\xi)\right)=\lambda d(\xi)$ ). Let $d$ be a homogeneous norm. There exist constants $C_{1}, C_{2}>0$ such that $C_{1}|\xi| \leq d(\xi) \leq$ $C_{2}|\xi|^{1 / r}$ for $d(\xi) \leq 1$, and $|\cdot|$ stands for the Euclidean norm.

Let $\tilde{\mu}$ be the matrix so that for any $i=1, \ldots, l$ it realizes the identity (2.3), that is, $X_{i}=\sum_{j=1}^{N} \tilde{\mu}_{i j}(\xi) \frac{\partial}{\partial \xi_{j}}$. It results that $\tilde{\mu}$ satisfies the assumption (2.4). Therefore, the matrix $\mu$ that realizes the identity $Y_{i}=\sum_{j=1}^{N} \mu_{i j}(\xi) \frac{\partial}{\partial \xi_{j}}$ also satisfies the assumption (2.4).

In [5, 8 it is proved that for any sub-laplacian $L$ there exists a homogeneous norm $d$ on $\mathbb{G}$ such that $\Gamma_{\eta}(\xi):=\left(d\left(\eta^{-1} \circ \xi\right)\right)^{2-Q}$ is a fundamental solution of $-L$ at $\eta$ (see also [2]).

In this setting, $Y_{i} d_{0}$ is homogeneous of degree 0 with respect to $\delta_{\lambda}$, hence $\left\|\psi_{\eta}\right\|_{\infty}=\left\|\psi_{0}\right\|_{\infty}$ by left invariance of $Y_{i}$. Thus, $\left(\mathbf{H}_{\eta}\right)$ and $\left(\mathbf{V}_{\eta}\right)$ hold for any point $\eta \in \mathbb{R}^{N}$. Therefore all the theorems of the previous section can be restated for sub-laplacians on Carnot groups and even for weak solutions (see Definition 2.17). 
Example 3.1. Simple examples of Carnot groups are the usual Euclidean spaces $\mathbb{R}^{Q}$. Moreover, if $Q \leq 3$, then $\mathbb{G}$ is the ordinary Euclidean space $\mathbb{R}^{Q}$.

The simplest nontrivial example of a Carnot group is the Heisenberg group $\mathbb{H}^{1}=$ $\mathbb{R}^{3}$. For an integer $n \geq 1$, the Heisenberg group $\mathbb{H}^{n}$ is defined as follows: Let $\xi=\left(\xi^{(1)}, \xi^{(2)}\right)=\left(x_{1}, \ldots, x_{n}, y_{1}, \ldots, y_{n}, t\right)=(x, y, t) \in \mathbb{R}^{2 n} \times \mathbb{R}$.

The Heisenberg group $\mathbb{H}^{n}$ is the set $\mathbb{R}^{2 n+1}$ endowed with the group law

$$
\hat{\xi} \circ \tilde{\xi}:=\left(\hat{x}+\tilde{x}, \hat{y}+\tilde{y}, \hat{t}+\tilde{t}+2 \sum_{i=1}^{n}\left(\tilde{x}_{i} \hat{y}_{i}-\hat{x}_{i} \tilde{y}_{i}\right)\right) .
$$

For $i=1, \ldots, n$, consider the vector fields

$$
X_{i}:=\frac{\partial}{\partial x_{i}}+2 y_{i} \frac{\partial}{\partial t}, Y_{i}:=\frac{\partial}{\partial y_{i}}-2 x_{i} \frac{\partial}{\partial t},
$$

and the associated Heisenberg gradient as follows:

$$
\nabla_{H}:=\left(X_{1}, \ldots, X_{n}, Y_{1}, \ldots, Y_{n}\right) .
$$

The Kohn laplacian $\Delta_{H}$ is then the operator defined by

$$
\Delta_{H}:=\sum_{i=1}^{n} X_{i}^{2}+Y_{i}^{2}
$$

The family of dilation is given by

$$
\delta_{\lambda}(\xi):=\left(\lambda x, \lambda y, \lambda^{2} t\right) .
$$

In $\mathbb{H}^{n}$ is defined the homogeneous norm

$$
|\xi|_{H}:=\left(\left(\sum_{i=1}^{n} x_{i}^{2}+y_{i}^{2}\right)^{2}+t^{2}\right)^{1 / 4} .
$$

The homogeneous dimension is $Q=2 n+2$ and the fundamental solution of the sub-laplacian $-\Delta_{H}$ at point $\eta$ is $\Gamma_{\eta}(\xi)=\left|\eta^{-1} \circ \xi\right|_{H}^{-2 n}$.

In what follows $L$ denotes a sub-laplacian on $\mathbb{G}$.

Theorem 3.2. Let $u$ be a weak solution of

$$
-L u(\xi) \geq f(\xi, u(\xi)) \quad \text { a.e. on } \mathbb{G} \text {. }
$$

If one of the following conditions is satisfied:

(1) There exist $p>1, C_{f}>0$ such that

$$
f(\xi, t) \geq C_{f}|t|^{p} \quad \text { for any } t \in \mathbb{R} \text { and a.e. } \xi \in \mathbb{G} ;
$$

(2) $f$ is nonnegative and (2.14) holds for some Lebesgue point $\eta_{0}$ of $u$ (and hence for all $\eta \in \mathbb{G}$ ),

then for any Lebesgue point $\eta$ of $u$ we have

$$
u(\eta) \geq \int_{\mathbb{R}^{N}} \Gamma_{\eta}(\xi) f(\xi, u(\xi)) d \xi .
$$

In particular, if in (3.49) the equality sign holds, then

$$
u(\eta)=\int_{\mathbb{R}^{N}} \Gamma_{\eta}(\xi) f(\xi, u(\xi)) d \xi .
$$


In order to prove the above theorem, it is sufficient to prove the following

Lemma 3.3. Let $u$ be a weak solution of (3.49) and let $\eta$ be its Lebesgue point. Then (2.19) holds.

Proof. This proof is similar to the proof of Lemma 2.18 in the Euclidean setting, so we shall be brief. Using the same notation, we have

$$
-I_{1}^{\epsilon}=(Q-2)(Q-2+m)\left(u \tilde{\phi} * \tilde{g}_{\epsilon}\right)(\eta),
$$

where $\tilde{g}_{\epsilon}(\xi):=\frac{1}{\epsilon^{Q}} \tilde{g}_{1}\left(\frac{x}{\epsilon}\right), \tilde{g}_{1}(\xi):=\psi_{\eta}^{2} \frac{d(\xi)^{m-2}}{\left(1+d(\xi)^{m}\right)^{\frac{Q-2}{m}+2}}$ and the convolution is defined as usual in a group. Using the analogue tools of Euclidean framework (see 6, 7, 9]), we concludes the proof.

A consequence of Theorem 2.8 is the following

Theorem 3.4. Assume that there exists $C_{f}>0,0 \leq p \leq 1$, such that

$$
f(\xi, t) \geq C_{f}|t|^{p} \quad \text { for any } t \in \mathbb{R} \text { and a.e. } \xi \in \mathbb{G} \text {. }
$$

Then (3.49) has no bounded nontrivial $\mathscr{C}^{1}$-weak solution.

3.1. Grushin type operators. Let $x \in \mathbb{R}^{d}, y \in \mathbb{R}^{k}$ and $N=d+k$. Consider the vector field $\nabla_{\gamma}$ given by $\sigma \nabla$ where $\sigma$ is the matrix

$$
\sigma:=\left(\begin{array}{cc}
I_{d} & 0 \\
0 & |x|^{\gamma} I_{k}
\end{array}\right)
$$

and $\gamma>0$. The second order differential operator associated to this vector field is the so-called Grushin type operator: $L=\Delta_{x}+|x|^{2 \gamma} \Delta_{y}$. For this operator it is not possible to endow $\mathbb{R}^{N}$ with a group law such that $\nabla_{\gamma}$ (and hence $L$ ) results to be left-invariant. The fundamental solution of $-L$ at the origin is given by

$$
\Gamma_{0}:=C_{d, k, \gamma}\left(|x|^{2+2 \gamma}+(1+\gamma)^{2}|y|^{2}\right)^{\frac{2-N-\gamma k}{2+2 \gamma}}=C_{d, k, \gamma} \llbracket \xi \rrbracket^{2-Q},
$$

where $Q:=N+k \gamma>2$ and $C_{d, k, \gamma}$ is a suitable positive constant (see [1, 4]). In this case $\psi_{0}:=\left|\nabla_{\gamma} \llbracket \xi \rrbracket\right|=|x|^{\gamma} / \llbracket \xi \rrbracket^{\gamma}$. It is easy to check that $\left(\mathbf{H}_{\eta}\right)$ and $\left(\mathbf{V}_{\eta}\right)$ hold for $\eta=0$. Therefore, Theorems 2.2, 2.6, 2.8, 2.12, and 2.22 hold with $\eta=0$.

Moreover, by the invariance of the operator $L$ under translation with respect to $y$, all the cited results hold with $\eta=(0, z) \in \mathbb{R}^{d} \times \mathbb{R}^{k}$.

Actually, by slight variation of the proof of Lemma 2.16, it is possible to prove that the representation formulae hold at point $(0, z)$ for weak solutions which are continuous at that point.

\section{REFERENCES}

[1] W. Beckner, On the Grushin Operator and Hyperbolic Symmetry, Proc. Am. Math. Soc. 129 (2001), 1233-1246. MR.1709740 (2001g:35009)

[2] A. Bonfiglioli, E. Lanconelli, F. Uguzzoni, Uniform Gaussian Estimates of the Fundamental Solutions for Heat Operators on Carnot Groups, Adv. Differential Equations 7 (2002), 1153-1192. MR1919700 (2003f:35054)

[3] H. Brezis, S. Kamin, Sublinear Elliptic Equations in $\mathbb{R}^{N}$, Manuscripta Math. 74 (1992), 87-106. MR:1141779 (93f:35062)

[4] L. D'Ambrosio, S. Lucente, Nonlinear Liouville theorems for the Grushin and Tricomi operators, J. Differential Equations 193 (2003), 511-541. MR1998967 (2004g:35241)

[5] G.B. Folland, Subelliptic estimates and function spaces on nilpotent Lie groups, Arkiv för Mat. 13 (1975), 161-207. MR0494315 (58:13215) 
[6] G.B. Folland, E.M. Stein, Hardy spaces on Homogeneous Groups, Math. Notes, vol. 28, Princeton Univ. Press, Princeton, 1982. MR0657581 (84h:43027)

[7] G.B. Folland, A course in Abstract Harmonic Analysis, Studies in Advanced Mathematics, CRC Press, Boca Raton, 1995. MR 1397028 (98c:43001)

[8] L. Gallardo, Capacités, mouvement brownien et problème de l'épine de Lebesgue sur les groupes de Lie nilpotents, Lecture Notes in Math. 928 (1982), 96-120. MR0669065 (84a:60089)

[9] P. Hajlasz, P. Koskela, Sobolev met Poincaré, Mem. Amer. Math. Soc. 145, no. 688 (2000), $\mathrm{x}+101$ pp. MR.1683160 (2000j:46063)

[10] J. Heinonen, Calculus on Carnot Groups, in Fall School in Analysis (Jyväskylä, 1994), vol. 68, Ber. Univ. Jyväskylä Math. Inst. Jyväskylä, 1995, 1-31. MR1351042 (96j:22015)

[11] E. Mitidieri, S.I. Pohozaev, A Priori Estimates of Solutions to Nonlinear Partial Differential Equations and Inequalities and Applications, Proc. Steklov Institute of Mathematics 234 (2001), 1-375. MR.1879326

[12] E. Mitidieri, S.I. PohOzAev, Positivity property of solutions of some elliptic inequalities on $\mathbb{R}^{n}$, Doklady Math. 68 (2003), 339-344.

Dipartimento di Matematica, via E. Orabona 4, Università degli Studi di Bari, I-70125 BARI, ITALY

E-mail address: dambros@dm.uniba.it

Dipartimento di Scienze Matematiche, via A. Valerio 12/1, Università degli Studi di Trieste, I-34127 Trieste, Italy

E-mail address: mitidier@units.it

Steklov Institute of Mathematics, Gubkina Str. 8, 117966 Moscow, Russia

E-mail address: pohozaev@mi.ras.ru 